\title{
Skin melanoma imaging using ultrasonography: a literature review
}

\author{
Paweł Piłat ${ }^{1,2}$, Adam Borzęcki $^{2}$, Mieczysław Jazienicki $^{3}$, Dorota Krasowska ${ }^{1}$ \\ ${ }^{1}$ Chair and Department of Dermatology, Venereology and Paediatric Dermatology, Medical University of Lublin, Lublin, Poland \\ ${ }^{2}$ Non-Public Health Care Centre Med-Laser, Lublin, Poland
}

${ }^{3}$ Department of Surgical Oncology, Independent Public Clinical Hospital, Lublin, Poland

Adv Dermatol Allergol 2018; XXXV (3): 238-242

DOI: https://doi.org/10.5114/ada.2018.76211

\begin{abstract}
Melanoma is one of the most unpredictable tumours, as regards both morphology and the course of disease. It may have different clinical forms making it very difficult to diagnose. Therefore, it often surprises even the most experienced dermatologists. Despite numerous attempts, no efficient treatment of advanced stages have been elaborated, and early detection and removal of a skin lesion currently constitutes the most efficient treatment method. Diagnostics uses different types of dermoscopic techniques characterised by considerable sensitivity and specificity in detecting melanoma at an early developmental stage. A continuous development of medical technologies leads to improving many methods of skin lesion imaging. Ultrasonography is one of these methods, enabling evaluation of the thickness of melanoma in vivo with high precision. Change thickness is the decisive factor influencing 5-year survival. The paper evaluates the usefulness of high-frequency ultrasonography (HFUS) in pre-operative melanoma imaging on the basis of literature review. Additionally, the use, role and placement of HFUS in skin melanoma diagnostics is determined.
\end{abstract}

Key words: melanoma, high-frequency ultrasonography, melanoma thickness, pre-procedure evaluation, margins.

\section{Introduction}

Currently, an increasing skin melanoma incidence is observed. The highest ratio of deaths in newly diagnosed cases in Europe is reported in the countries of Central and Eastern Europe [1]. Patients with suspected skin melanoma are subjected to clinical examination and dermoscopic examination. In recent years, a substantial advancement of pigmented mole examination methods has been achieved, and the dermatoscope is currently used not only for the evaluation of tumours, but also in diagnosis of numerous skin diseases. In recent decades, a dynamic progress of dermoscopy has taken place, both in the field of devices used and the diagnostic algorithm method. The model analysis developed by Kittler et al. [2] appears to be nowadays the most advanced and complex method. On the basis of dermatoscopic examination, the physician decides on subsequent treatment. Potentially neoplastic changes are removed and the obtained preparation is subjected to histological assessment. The key parameter for histological assessment of the lesion is thickness expressed in millimetres and provided using the Breslow scale [3]. Further decisive parameters include the number of mitoses, presence of ulceration, number of affected lymph nodes, satellitoses and distant metastases $[4,5]$. On the basis of results obtained, a decision is made on the possible additional surgical excision, sentinel lymph node biopsy or beginning systemic treatment $[6,7]$. Patients from the group of elevated risk should be constantly monitored by appropriate specialists, depending on the advancement stage of the disease [8-10]. It is known that correct determination of the excision margin and also its depth are decisive for the early stage of diagnostics and treatment. The optimal situation consists of a radical excision of the change with a correct margin of unaffected skin and also subcutaneous tissue, adequately to the level of malignancy [10-12]. It appears that high-frequency ultrasonography (HFUS) examination, as a repetitive, non-invasive, and painless examination, may play a key role in the pre-operative in vivo skin melanoma evaluation and it may correlate with the result obtained in histopathological examination. Currently, HFUS examination is becoming more available in hospitals and at dermatology practices [13]. In the last 10 years, authors of numerous published studies attempted to evaluate

Address for correspondence: Paweł Piłat MD, Non-Public Health Care Centre Med-Laser, 8/68 Szeligowskiego St, 20-883 Lublin, Poland, fax: +48 607760 179, e-mail: pawelpilat1@gmail.com

Received: 11.03.2017, accepted: 23.07.2017. 
melanoma thickness using ultrasound. Results of these studies provide answers to many questions and also inspire to ask a great number of new ones.

The objective of the present paper was to evaluate applicability of HFUS examination for pre-operative assessment of melanoma on the basis of a literature review.

\section{High-frequency ultrasonography in skin tumours}

Ultrasonography (USG) examination is based on the phenomenon of ultrasound wave propagation in elastic media. Thanks to that, it is possible to highlight boundary surfaces of different types of tissues and organs, which in turn enables determination of the depth of the evaluated changes. The key parameter influencing quality of the obtained image is the resolution of the device. It is divided into three types: axial, lateral and contrast. The resolution consisting of the smallest distance between two points (positioned in one axis) of the tested tissue, expressed in millimetres is the key element of the measurement of thickness and depth of skin changes. However, generally it is better than axial lateral resolution and it increases with the increase of the head frequency. Typical ultrasonographic image of healthy skin consists of three elements - epidermis, also referred to as epidermal echo, dermis and subcutaneous tissue.

First attempts at using USG for skin imaging were made by Alexander and Miller over 40 years ago, using a $15 \mathrm{MHz}$ head [14]. Since that time, a considerable technological advancement has taken place, which enabled an increase in head frequencies, which lead to an increase in the obtained image resolution [15]. This makes it possible to do diagnostic tests of the entire change in real time, without violation of the skin integrity and obtaining clear images, and even evaluation of the skin blood flow. Currently, HFUS is used for diagnosis and treatment monitoring of numerous diseases, including localised scleroderma, lichen sclerosus, psoriasis limited to the skin and nails, and also skin tumours [16, 17]. A study was described, which involved sonographies of 4338 skin changes included in 8 groups: benign nonvascular tumours, benign vascular tumours, malignant tumours, vascular non-cancerous changes, articular changes and periarticular changes, inflammatory and infectious changes, skin appendages, and nail apparatus. The conducted HFUS improved the preliminary diagnosis accuracy from $73 \%$ to $97 \%$ ( $p<0.01)$. The examination sensitivity was $99 \%$ and specificity - 100\% [18]. In the majority of cases, in the HFUS examination melanoma appears as a hypoechoic area similar to a biconvex lens, normally located at the dermal/epidermal junction. This image may differ depending on the thickness of the melanoma, presence of ulceration and its location. Currently, histopathological examination is the golden standard for melanoma diagnosis.
An expert team elaborated detailed guidelines for the microscopic evaluation of melanoma [19]. In recent years, numerous papers have been published determining the applicability of sonographic examination for pre-operative evaluation of this type of changes, and the obtained results showed a correlation of melanoma thickness in ultrasonographic examination and histopathological examination.

Jasaitiene et al. combined data from 22 studies on the measurement of melanoma thickness using ultrasonography. After an analysis of detailed results, the authors determined the applicability of USG and HFUS examinations and pointed out to the high index of correlation between the change thickness evaluated in histopathological examination and that obtained in USG and HFUS examinations ( $r$ in the range from 0.887 to 0.99). In the HFUS examination, melanoma is visible as a homogenous, well-marked area. Authors of the paper particularly emphasise the applicability of HFUS examination in the pre-operative evaluation of melanoma, particularly for the selection of correct excision margins, as well as the conduct of biopsy of the sentinel lymph node during one procedure. This limits the stress to which patient is exposed and decreases treatment costs, accelerates diagnostic and treatment procedures [20].

Table 1 presents results of studies published in 20102014 on the correlation of melanoma thickness obtained in sonometry and in histopathological examination.

Music et al. provided two experienced radiologists with images obtained in an examination with $12-15 \mathrm{MHz}$ head, for evaluation. The obtained results demonstrated that USG examination is characterised by high frequency (92\%), specificity (92\%), positive (95\%) and negative (81\%) predictive value and the level of correlation between histopathological examination and sonographic examination was determined as very high $(r=0.823$, $p<0.001)$. However, it is strongly emphasised that these values referred to melanomas thicker than $1 \mathrm{~mm}$. To evaluate usability of HFUS examination in $>1 \mathrm{~mm}$ melanoma assessment, the authors emphasise the necessity to conduct a larger number of tests [21].

Similar analysis was conducted by Kaikaris et al. On the basis of a study conducted with the use of a $14 \mathrm{MHz}$ head, the authors believe that overinterpretations of melanoma thickness are numerous, which is caused by the accumulation of lymphocytes in the lower area of the tumour. In the case of the used frequencies, a decision on treatment and possible sentinel lymph node biopsy (SLNB) should be taken for melanomas with a thickness greater than $2 \mathrm{~mm}$. If changes have a lower thickness, a re-examination with a $100 \mathrm{MHz}$ head or excisional biopsy should be performed, and on the basis of the histopathological result obtained, an additional excision and sentinel lymph node biopsy should be carried out [22]. It also appears that the considerable constriction of the preparation after its excision and placement in formalin 
Table 1. Correlation between thickness measurement of the skin tumours obtained in histopathological and ultrasonographic examination (2010-2014)

\begin{tabular}{lccc}
\hline Researcher & $\begin{array}{c}\text { Head frequency } \\
f[\mathrm{MHz}]\end{array}$ & $\begin{array}{c}\text { Melanomas examined } \\
n\end{array}$ & $\begin{array}{c}\text { Correlation level } \\
r\end{array}$ \\
\hline Music et al., 2010 [21] & $12-15$ & 70 & 0.823 \\
\hline Kaikaris et al., 2011 [22] & 14 & 100 & 0.283 for T 1-2 mm \\
& & & 0.869 for T $2 \mathrm{~mm}$ \\
\hline Hinz et al., 2011 [24] & 20 & 28 & Not given \\
\hline Crisan et al., 2013 [27] & 20 & 79 & 0.994 \\
\hline $\begin{array}{l}\text { Fernández Canedo et al., 2013 [26] } \\
\text { (retrospective study) }\end{array}$ & 15 & 67 & 0.984 \\
\hline \begin{tabular}{l} 
Meyer et al., 2014 [25] \\
\hline
\end{tabular} & 25 & & 0.678 \\
\hline
\end{tabular}

may influence the overestimation of the change thickness. This stems from the structure and placement of collagen fibres, and also from the effect of formalin on human tissues. As a result of this, higher values of thickness are provided after histopathological examination than those obtained in sonographic examination [20, 23].

Hinz et al. published a study which determined the precision of skin pigment change thickness evaluation using 1325-nm optical coherence tomography (OCT). Additionally, they compared obtained results with values obtained in histopathological and sonographic examinations. Changes, thickness of which was greater than $1 \mathrm{~mm}$ were excluded from the study due to the dispersion present in the OCT examination. Change image evaluated using OCT was characterised by lower contrast with surrounding tissues in comparison to that obtained using HFUS. A decision on the selection of the appropriate healthy tissue margin during the removal of suspected malignant changes was taken on the basis of result obtained using sonometry. Measurements were conducted between the epidermal echo peak or its midpoint, and the lowest part of the hypoechoic area, which might have considerably overstated melanoma thickness. The majority of researchers measure changes from the lowest part of the epidermal echo. Authors emphasise that HFUS heads with 20-50 MHz frequency are usable for pre-operative evaluation of pigment changes, however, studies using $100 \mathrm{MHz}$ frequency heads are necessary. Results of the study indicate the advantage of OCT over $20 \mathrm{MHz}$ HFUS in the case of melanomas with thickness not exceeding $1 \mathrm{~mm}$ [24].

An interesting study was published by Meyer et al. which compared the usability of the melanoma thickness evaluation using HFUS (25 MHz) and 930-nm OCT. The study was conducted on 131 patients, which had at least one suspicious pigment change by two independent dermatologists trained in the use of HFUS and OCT. Changes were evaluated two times at 7-day intervals. On the basis of the conducted study, the authors determined that OCT was not precise enough for melanoma thickness evaluation (ICC = 0.0), and disparity with the histopathological examination result increased with the increase of change depth, particularly over $0.5 \mathrm{~mm}$. Furthermore, the OCT examination lasted much longer than the sonographic examination. Authors of the study obtained a good correlation of results obtained in HFUS examination with histopathological examinations [25].

Fernández Canedo et al. carried out retrospective comparative analysis of parameters obtained using 15 MHz USG and histopathological examination of 79 changes of primary melanoma character. They assessed thickness, presence of ulceration, regression, and inflammatory infiltrate. It turned out that the sonographic method with determined thickness of superficial melanoma forms with high precision, which enabled precise determination of excision margins. The authors emphasised low usability of the method in evaluation of nodular and vertically spreading changes. No significant relationships between parameters other than thickness could be determined, which were established in microscope and sonographic examination [26].

Crisan et al. presented results obtained in a study using a $14 \mathrm{MHz}$ Colour Doppler head and a $20 \mathrm{MHz}$ head. With the use of a $14 \mathrm{MHz}$ head, the authors determined a lack of flows in melanoma type lesions spreading superficially $(n=8)$ and rich vascularity in nodular lesions $(n=20)$. In the case of a $20 \mathrm{MHz}$ head, the authors point out to the underestimation of nodular melanoma thickness and overestimation of superficially spreading melanomas in comparison to a histopathological examination, however, the high level of correlation between sonographic and histopathological measurements is retained (> 98\%). Moreover, the study evaluated basal cell carcinomas and on the basis of the obtained results a four-stage Breslow scale of advancement for basal cell carcinoma was elaborated for HFUS examination. On the basis of the obtained sonographic measurements, the 
authors identified stage I: < $0.8 \mathrm{~mm}$, stage II: 0.8-1.88 $\mathrm{mm}$, stage III: $1.88-3 \mathrm{~mm}$, and stage IV: $>3 \mathrm{~mm}$ [27].

\section{Other methods for melanoma imaging}

Different procedures are performed at an early diagnostic stage. The diagnostics of pigment changes utilises monitoring and mapping tools, dermatoscopes and videodermatoscopes, which enable evaluation of the morphology of pigment changes. On the other hand, confocal microscopes enable evaluation of skin lesions at a cellular level. Individual methods are used in the case of screening in patients with an elevated carcinogenesis risk or for those, which were diagnosed with skin melanoma. Concomitant use of the mentioned methods improves efficacy in early diagnosis and differential diagnostics of melanoma [28].

There are papers evaluating melanoma vascularization using Colour Doppler ultrasonography. Lassau et al. emphasise the fact that vessels can be highlighted in lesions thicker than $2 \mathrm{~cm}$, which seems to indicate their greater malignancy [29]. It appears that the presence of vessels depends on the clinical form of melanoma, in nodular lesions it is possible to image the flow in the vessels [25].

An ongoing research is conducted on the expansion of non-invasive imaging of melanoma and its metastases. Methods such as ultrasonography, computed tomography (CT), positron emission tomography (PET) or a combination of both (PET-CT) are used for this purpose. Results published thus far indicate the advantage of ultrasonography of the sentinel lymph node over other methods of the search of metastases. On the other hand, PET-CT turned out to be the best examination for the detection of distant metastases [30].

High-frequency ultrasonography is often used for differential diagnostics of skin lesions. Characteristics are looked for in a sonographic image for changes such as pigmented moles, dermatofibromas, seborrheic warts, angiomas, blue naevi, basal cell carcinomas, melanoma metastases to skin [31-33]. It is worth emphasising that HFUS examination is reimbursed by health insurance in numerous countries of Western Europe, such as France and Germany [33].

\section{Summary}

The use of HFUS examination for the evaluation of cancerous changes on the skin provides a considerable amount of information and significantly improves the diagnostic and therapeutic process. Evaluation of the thickness of melanoma seems to be the most important element of HFUS examination, which doubtlessly helps making a decision on excision of the change with a margin adequate for the thickness of the melanoma. However, the development of standardisation of the method appears indispensable so that it can be better used in the decision on the melanoma excision margin.

\section{Conflict of interest}

The authors declare no conflict of interest.

\section{References}

1. Forsea AM, Marmol V, Vries E, et al. Melanoma incidence and mortality in Europe: new estimates, persistent disparities. Br J Dermatol 2012; 167: 1124-30.

2. Kittler H, Rosendahl C, Cameron A, Tschandl P. Dermatoscopy. Algorithmic Method Based on Pattern Analysis. Via Medica, Gdansk 2012.

3. Balch CM, Gerschenwald JE, Soong SJ, et al. Final version of 2009 AJCC melanoma staging and classification. J Clin Oncol 2009; 27: 6199-206.

4. Fong ZV, Tanabe KK. Comparison of melanoma guidelines in the U.S.A., Canada, Europe, Australia and New Zealand: a critical appraisal and comprehensive review. Br J Dermatol 2014; 170: 20-30.

5. Nagore E, Oliver V, Botella-Estrada R, et al. Prognostic factors in localised invasive cutaneous melanoma: high value of mitotic rate, vascular invasion and microscopic satellitosis. Melanoma Res 2005; 15: 169-77.

6. Morton DL, Cochran AJ, Thompson JF. The rationale for sentinel-node biopsy in primary melanoma. Nat Clin Pract Oncol 2008; 5: 510-11.

7. Sondak VK, Gibney GT. Indications and options for systemic therapy in melanoma. Sure Clin North Am 2014; 94: 1049-58.

8. Watts CG, Dieng M, Morton RL, et al. Clinical practise guidelines for identification, screening and follow-up of individuals at high risk of primary cutaneous melanoma: a systematic review. Br J Dermatol 2015; 172: 33-47.

9. Mrazek AA, Chao C. Surviving cutaneous melanoma: a clinical review of follow-up practises, surveillance, and management of recurrence. Surg Clin North Am 2014; 94: 989-1002.

10. Mayer JE, Swetter SM, Fu T, et al. Screening, early detection, education, and trends for melanoma: current status (2007-2013) and future directions: Part II. Screening, education, and future directions. I Am Acad Dermatol 2014; 71: 611.e1-10.

11. Rawlani R, Rawlani V, Qureshi HA, et al. Reducing margins of wide local excision in head and neck melanoma for function and cosmesis: 5-year local recurrence-free survival. J Surg Oncol 2015; 111: 795-9.

12. Sharma KS, Lim P, Brotherstoni MT. Excision versus incision biopsy in the management of malignant melanoma. J Dermatolog Treat 2016; 27: 88-90.

13. Polańska A, Dańczak-Pazdrowska A, Jałowska M, et al. Current applications of high-frequency ultrasonography in dermatology. Adv Dermatol Allergol 2017; 34: 535-42.

14. Alexander H, Miller DL. Determining skin thickness with pulsed ultra sound. J Invest Dermatol 1979; 72: 17-9.

15. Mlosek RK, Malinowska S. Ultrasound image of the skin, apparatus and imaging basics. J Ultrason 2013; 13: 2012-21.

16. Szymańska E, Nowicki A, Mlosek K, et al. Skin imaging with high frequency ultrasound - preliminary results. Eur J Ultrasound 2000; 12: 9-16.

17. Mihaela EM, Jid CB, Roman II, et al. Ultrasonography in psoriatic disease. Med Ultrasound 2015; 17: 377-82. 
18. Wortsman X, Wortsman J. Clinical usefulness of variablefrequency ultrasound in localised lesions of the skin. J Am Acad Dermatol 2010; 62: 247-56.

19. Scolyer RA, Judge MJ, Evans A, et al. Data set for pathology reporting of cutaneous invasive melanoma: recommendations from the International Collaboration on Cancer Reporting (ICCR). Am J Surg Pathol 2013; 37: 1797-814.

20. Jasaitiene D, Valiukeviciene S, Linkeviciute G. Principles of high-frequency ultrasonography for investigation of skin pathology. J Eur Acad Dermatol Venereol 2011; 25: 375-82.

21. Music MM, Hertl K, Kadivec M, et al. Pre-operative ultrasound with a $12-15 \mathrm{MHz}$ linear probe reliably differentiates between melanoma thicker and thinner than $1 \mathrm{~mm}$. J Eur Acad Dermatol Venereol 2010; 24: 1105-8.

22. Kaikaris V, Samsanavičius D, Maslauskas K, et al. Measurement of melanoma thickness: comparison of two methods: ultrasound versus morphology. J Plast Reconstr Aesthet Surg 2011; 64: 796-802.

23. Blasco-Morente G, Garrido-Colmenero C, Pérez-López I, et al. Study of shrinkage of cutaneous surgical specimens. J Cutan Pathol 2015; 42: 253-7.

24. Hinz T, Ehler L, Voth H, et al. Assessment of tumour thickness in melanocytic skin lesions: comparison of optical coherence tomography, 20-MHz ultrasound and histopathology. Dermatology (Basel) 2011; 223: 161-8.

25. Meyer N, Lauwers-Cances V, Lourari S, et al. High-frequency ultrasonography but not 930-nm optical coherence tomography reliably evaluates melanoma thickness in vivo: a prospective validation study. Br I Dermatol 2014; 171: 799-805.

26. Fernández Canedo I, de Troya Martín M, Fúnez Liébana R, et al. Preoperative 15- $\mathrm{MHz}$ ultrasound assessment of tumour thickness in malignant melanoma. Actas Dermosifiliogr 2013; 104: 227-3.

27. Crisan M, Crisan D, Sannino G, et al. Ultrasonographic staging of cutaneous malignant tumours: an ultrasonographic depth index. Arch Dermatol Res 2013; 305: 305-13.

28. Wang SQ, Hashemi P. Noninvasive imaging technologies in the diagnosis of melanoma. Semin Cutan Med Surg 2010; 29: 174-84.

29. Lassau N, Lamuraglia M, Koscielny S, et al. Prognostic value of angiogenesis evaluated with high-frequency and colour Doppler sonography for preoperative assessment of primary cutaneous melanomas: correlation with recurrence after a 5 year follow-up period. Cancer Imaging 2006; 25: 24-9.

30. Xing Y, Bronstein Y, Ross MI, et al. Contemporary diagnostic imaging modalities for the staging and surveillance of melanoma patients: a meta-analysis. J Natl Cancer Inst 2011; 103: 129-42.

31. Samimi M, Perrinaud A, Naouri M, et al. High-resolution ultrasonography assists the differential diagnosis of blue naevi and cutaneous metastases of melanoma. Br J Dermatol 2010; 163: 550-6.

32. Badea R, Crişan M, Lupşor M, et al. Diagnosis and characterization of cutaneous tumours using combined ultrasonographic procedures (conventional and high resolution ultrasonography). Med Ultrason 2010; 12: 317-22.

33. Machet L, Samimi M, Georgesco G, et al. High resolution ultrasound imaging of melanocytic and other pigmented lesions of the skin. In: Ultrasound imaging. Tanabe M (ed.) InTech, DOI: 10.5772/15372. Available from: http://www.intechopen.com/books/ultrasound-imaging/high-resolutionultrasound-imaging-of-melanocytic-and-other-pigmentedlesions-of-the-skin. 